\title{
Thermal background fluctuations at 10 micron measured with VLTI/MIDI
}

\author{
Olivier Absil ${ }^{a}$, Eric J. Bakker ${ }^{b}$, Markus Schöller ${ }^{c}$ and Philippe Gondoin ${ }^{d}$ \\ ${ }^{a}$ Institut d'Astrophysique et de Géophysique, 17 Allée du Six Août, 4000 Liège, Belgium \\ ${ }^{b}$ Leiden Observatory, P.O. Box 9513, 2300 RA Leiden, The Netherlands \\ ${ }^{c}$ European Southern Observatory, Casilla 19001, Santiago 19, Chile \\ ${ }^{d}$ ESTEC, European Space Agency, P.O. Box 299, 2200 AG Noordwijk, The Netherlands
}

\begin{abstract}
We present an experiment to measure the thermal background level and its fluctuations with the European Southern Observatory (ESO) Very Large Telescope Interferometer (VLTI). The Mid Infrared Instrument (MIDI) operating between 8 and 12 micron was used in both dispersed and non-dispersed modes. By using an interferometric instrument, in non-interferometric mode, we probe the same optical path as can be expected for other infrared interferometric instruments, e.g. GENIE and MIDI itself. Most of the infrared thermal background detected with MIDI originates from the VLTI infrastructure. This can be attributed to the absence of a pupil re-imaging mirror. Only for a small region around the optical axis of the system the signal from the VLTI infrastructure can be considered small and the atmospheric background fluctuations can be characterized.

The fluctuations of the thermal emission are described in terms of their power spectral densities (PSD). We have identified two regions in the PSD. For the low frequency range $(0-10 \mathrm{~Hz})$ the fluctuations are dominated by the Earth atmosphere. The slope of the log-log PSD is close to -1 . For the high frequency (larger than $10 \mathrm{~Hz}$ ) range the fluctuations are due to photon noise and the PSD flattens off. Many narrow peaks are present in the PSD. Peaks at 1 and $50 \mathrm{~Hz}$ occur in almost all data sets and are identified as the effects of the MIDI closed cycle cooler and the power lines respectively. Other peaks at 10 and $30 \mathrm{~Hz}$, as well as peaks above $50 \mathrm{~Hz}$, are assumed to be VLTI or MIDI-specific frequencies.
\end{abstract}

Keywords: Interferometry, infrared, MIDI, GENIE, thermal background, turbulence

\section{INTRODUCTION}

Interferometric observations at wavelengths between $3 \mu \mathrm{m}$ and $25 \mu \mathrm{m}$ are background limited. This background radiation has the largest contribution at $10 \mu \mathrm{m}$. With the exception of very strong astronomical sources the signal from the thermal background emitted by the Earth atmosphere and the telescope is larger than the signal from the astronomical sources. This background signal changes unpredictably on timescales of milli-seconds due to turbulence in the air. Atmospheric blobs at different temperatures and relative humidity passing through the optical path are causing both absorption of the signal and additional emission. In almost all cases scientific instruments operating beyond $3 \mu \mathrm{m}$ must correct for the background radiation through chopping at a frequency typically between 5 and $20 \mathrm{~Hz}$.

At the Very Large Telescope Interferometer (VLTI) two instruments have to compensate for background radiation: the Mid-Infrared Instrument (MIDI, Ref. 1) and the Ground-based European Nulling Interferometry Experiment (GENIE, Ref. 2). In this paper we present the results of a series of experiments conducted to characterize the thermal background at the VLTI. We have used the only available mid-infrared instrument that sees the full optical path through the interferometer. This instrument operates between 8 and $12 \mu \mathrm{m}$ and can be used with a filter to see a full image or with a dispersive element (low-resolution prism or high-resolution grism) in combination with a slit to record a dispersed image. The result of our experiments will help decide on the specific parameters to be used for chopping by MIDI and GENIE (e.g. chopping frequency) and whether a more advanced method for background subtraction should be considered.

Send correspondence to absil@astro.ulg.ac.be or bakker@strw.leidenuniv.nl. 
We expect several components in the power spectrum of the background radiation (Ref. 3-5): the shot noise of the photon flux itself and a " $1 / \mathrm{f}$ " noise due to variations in the background caused by temperature drifts and clouds. Additionally we expect read noise, " $1 / \mathrm{f}$ " noise, and a slow drift in sensitivity of the detector. Fluctuations comparable to a Kolmogorov spectrum would give a slope of $-8 / 3$ and those comparable to a random intensity walk to a slope of -2 (see details in Ref. 6). For scientific observations we would like to remove all these effects and aim at reaching Background-Limit Performance (BLIP) where the photon noise dominates the other sources.

\section{OBSERVATIONS}

Measurements of the thermal background of the VLTI with MIDI have been performed on two occasions.

\subsection{February 2003}

The first set of data was obtained during commissioning of MIDI (18 to 24 February 2003) with UT1-UT3 and SD1-SD2 (Table 1). Data was collected in non-dispersed mode using filters (broad-band N, and narrow band N11.3 and N8.7). Typical frame integration times are 1.2 milliseconds (msec) for the broad-band filter, and $4.0 \mathrm{msec}$ for narrow-band filters. The optical path between the telescopes and the MIDI instrument was not yet optimally aligned. This led to some vignetting of the optical beam by mirror mounts, etc. In addition to this, there was no pupil relay optics available during this experiment. The effect of this is that vignetting of the beams does not only remove part of the light, but the components responsible for the vignetting also emit thermal radiation into the optical path. Since the VLTI infrastructure emits at a temperature significantly warmer than the cold sky (and with a higher efficiency) these observations are very much affected by the absence of pupil transfer optics. For the October 2003 experiment the optical train was much better aligned. This improvement of pupil plane imaging is demonstrated by comparing a pupil plane image recorded in February 2003 (Fig. 1(a)) to one recorded in October 2003 (Fig. 1(b)).

\subsection{October 2003}

The second data-set was recorded during an observing run between 3 to 10 October 2003 using a single telescope (UT3) (Table 1). This time MIDI was operated in dispersed mode (PRISM mode, $R \approx 30$ ) with a slit of $0.2 \mathrm{~mm}$. The light was focused on the detector by the field camera lens. Since only one telescope was used only a single window of the MIDI detector was read-out and stored. The lower corner of this window was $(\mathrm{x}, \mathrm{y})=(1,141)$ with a size of $(\delta \mathrm{x}, \delta \mathrm{y})=(320,50)$. The main delay line number 3 was used at a fixed position of 6 meters to have the best pupil matching within the delay tunnel. During the observations no active control of the pupil was available (no variable curvature mirrors in place yet). Therefore the thermal emission from the VLTI infrastructure has affected the measurements. The detector integration time per frame was $2 \mathrm{msec}$, corresponding to a cycle time (integration, read-out, reset) of $6 \mathrm{msec}$. A typical detector count rate was 10000 counts/pixel/frame (saturation occurs at 65000 , non-linearity starts at 50000). The main difference between this October 2003 and the previous February 2003 experiment was that the VLTI optical train was much better aligned, the main delay lines were positioned at a location which gives the best pupil plane, and MIDI was able to operate in dispersed mode. This latter allows us to study the thermal background characteristics in much greater details than before.

\section{DATA ANALYSIS}

\subsection{Characterization of thermal background characteristics}

Turbulent behavior is characterized through a power spectral density (PSD) analysis. In essence, it makes the Fourier Transform of a time series. For each frequency in the PSD, it computes the amplitude and phase needed to characterize the time series. The PSD is plotted as power (square of the amplitude) versus frequency. If the $\mathrm{y}$-axis units of the time series are "ADU/pixel/DIT", the units of the PSD are "(ADU/pixel/DIT $)^{2} / \mathrm{Hz}$ ".

The intensity of the pixels within a region are averaged (yielding an average intensity per pixel for that region), and divided by the detector integration time (to be independent of the integration time). With a typical count rate of $10^{4} \mathrm{ADU} / \mathrm{pixel} / \mathrm{DIT}$, and a typical DIT of $4 \mathrm{~ms}$, a typical count rate of $2.5 \times 10^{6} \mathrm{ADU} / \mathrm{pixel} / \mathrm{second}$ is expected. The conversion from counts to electrons is a factor 145 (electrons per ADU) and a detector efficiency of 0.36 . Typical count rates are therefore $10^{9}$ photons/pixel/second. 
Table 1. Log of the thermal background fluctuation observations with MIDI. The file names contain the date and universal time of observation. DIT is the detector integration time in msec.

\begin{tabular}{|c|c|c|c|c|c|}
\hline Exp. \# & File & DIT & \# frames & Filter & Comments \\
\hline \multicolumn{6}{|c|}{ February 2003} \\
\hline 00 & 2003-02-18T06:57 & 4.0 & $3 \times 100000$ & N11.3 & MIDI black-screen \\
\hline 01 & 2003-02-19Т05:58 & 4.0 & 100000 & N11.3 & SD1, SD2 on Eta Carinae, DL trajectory \\
\hline 02 & 2003-02-19Т08:28 & 4.0 & $2 \times 100000$ & N11.3 & SD1, SD2 @ zenith, dome closed \\
\hline 03 & 2003-02-19Т09:31 & 4.0 & 100000 & N8.7 & SD1, SD2 @ zenith, dome closed \\
\hline 04 & 2003-02-20T06:37 & 1.2 & $35000+2 \times 40000$ & Nband & UT1 standing, UT3 tracking, on sky \\
\hline 05 & 2003-02-20Т07:30 & 1.2 & 41000 & Nband & UT1 standing, UT3 tracking, dome closed \\
\hline 06 & 2003-02-20T08:01 & 4.0 & $30000+100000$ & N8.7 & UT1 standing, UT3 tracking, dome closed \\
\hline 07 & 2003-02-20Т09:24 & 4.0 & 100000 & N8.7 & Black-plates in DL tunnel \\
\hline 08 & 2003-02-22Т06:32 & 1.2 & $3 \times 100000$ & Nband & $\begin{array}{l}\text { UT1 tracking, UT3 standing } 45^{\circ} \text { North, } \\
\text { DL fixed, dome closed }\end{array}$ \\
\hline 09 & 2003-02-22Т07:48 & 1.2 & $3 \times 100000$ & Nband & $\begin{array}{l}\text { UT1 and UT3@ zenith, dome closed, DLs } \\
\text { moving }\end{array}$ \\
\hline 10 & 2003-02-22Т09:17 & 1.2 & 70000 & Nband & Black-plate in DL, Delay Lines fixed \\
\hline 11 & 2003-02-23Т02:07 & 1.2 & 100000 & Nband & $\begin{array}{l}\text { UT1, UT3 on sky, people at UT3 Coudé, } \\
\text { only tracking, no guiding }\end{array}$ \\
\hline 12 & 2003-02-23Т02:25 & 1.2 & 100000 & Nband & $\begin{array}{l}\text { No people at UT3 Coudé, Z Canis Ma- } \\
\text { joris as source }\end{array}$ \\
\hline 13 & 2003-02-24Т09:52 & 1.2 & 100000 & Nband & $\begin{array}{l}\text { UT1 Zenith, UT3 } 45^{\circ} \text { North, DL1 }= \\
\text { DL3 }=7 \text { meters standing }\end{array}$ \\
\hline \multicolumn{6}{|c|}{ October 2003} \\
\hline \multirow[t]{8}{*}{20} & 2003-10-07T02:48 & 2 & $3 \times 100000$ & PRISM & Elevation of $90^{\circ}$ \\
\hline & 2003-10-07Т06:04 & 2 & $3 \times 100000$ & PRISM & Elevation of $80^{\circ}$ \\
\hline & 2003-10-07Т09:10 & 2 & $3 \times 100000$ & PRISM & Elevation of $70^{\circ}$ \\
\hline & 2003-10-07Т03:34 & 2 & $3 \times 100000$ & PRISM & Elevation of $60^{\circ}$ \\
\hline & 2003-10-07Т07:05 & 2 & $3 \times 100000$ & PRISM & Elevation of $50^{\circ}$ \\
\hline & not available & & & PRISM & Elevation of $40^{\circ}$ \\
\hline & 2003-10-07T04:58 & 2 & $3 \times 100000$ & PRISM & Elevation of $30^{\circ}$ \\
\hline & 2003-10-07Т08:00 & 2 & $3 \times 100000$ & PRISM & Elevation of $20^{\circ}$ \\
\hline
\end{tabular}

The extracted intensity $h_{j}\left(t_{j}\right)$ has units of $\mathrm{ADU} / \mathrm{pixel} / \mathrm{sec}$ and $t_{j}$ has units of seconds. Before computing the PSD, we remove the DC offset of the time series. This new signal is noted $V_{j}\left(t_{j}\right)$ and has units of ADU/pixel/sec. The total energy in $V_{j}$ is computed by

$$
E=\int_{t=0}^{t=\infty}|V(t)|^{2} d t=\sum_{j=0}^{N-1}\left|V_{j}\right|^{2} \cdot \Delta t
$$

with $\Delta t$ being the step size of the time series. The energy $E$ of the signal has units of $(\mathrm{ADU} / \mathrm{pixel} / \mathrm{second})^{2}$. For a simple sine signal $V_{j}=A \sin \left(2 \pi f t_{j}-\phi\right)$, this implies:

$$
E=\int_{t=0}^{t=T} A^{2} \sin ^{2}(2 \pi f t-\phi)=\frac{A^{2}}{2 \pi f} \int_{t=0}^{t=T} \sin ^{2} x d x
$$

which gives

$$
E=A^{2} \frac{T}{2}
$$

with $A$ the amplitude of the sine wave in ADU/pixel/sec, $T$ the total time span in sec, $f$ the frequency of the wave in $\mathrm{Hz}$ and $\phi$ the phase offset in radians. The power $P$ of the signal is defined as the energy per second, i.e., 


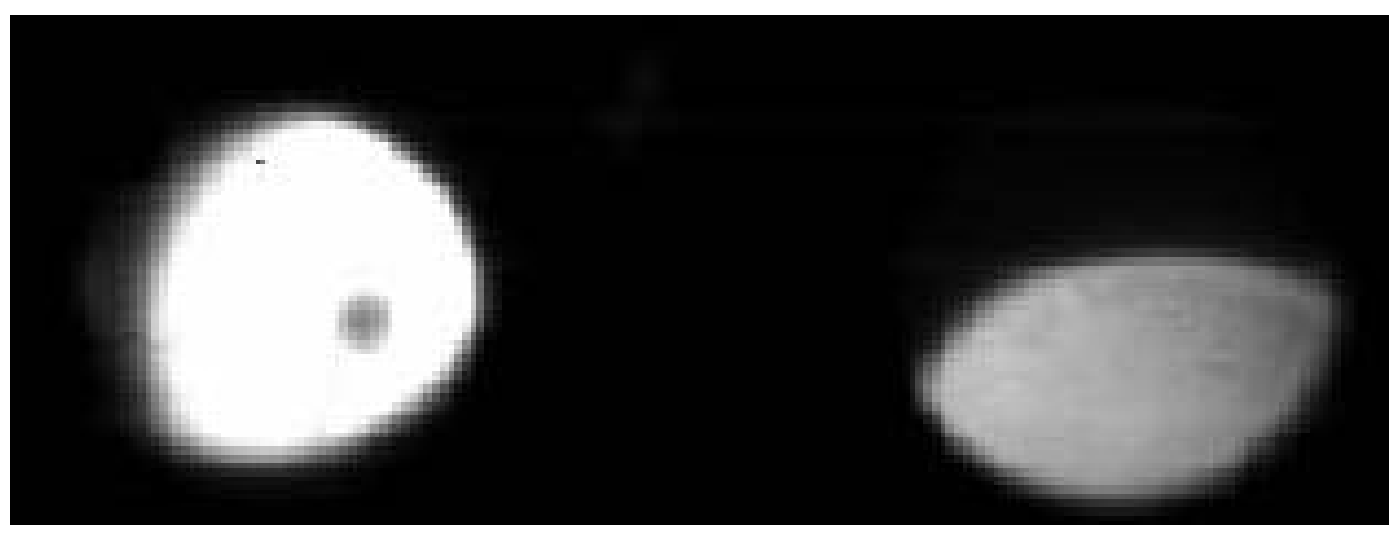

(a) Vignetting of the pupil plane by MIDI during the February 2003 experiment. Left image is UT3 (brightest spot, MIDI beam B), right image is UT1 (MIDI beam A). The different shape is due to vignetting.

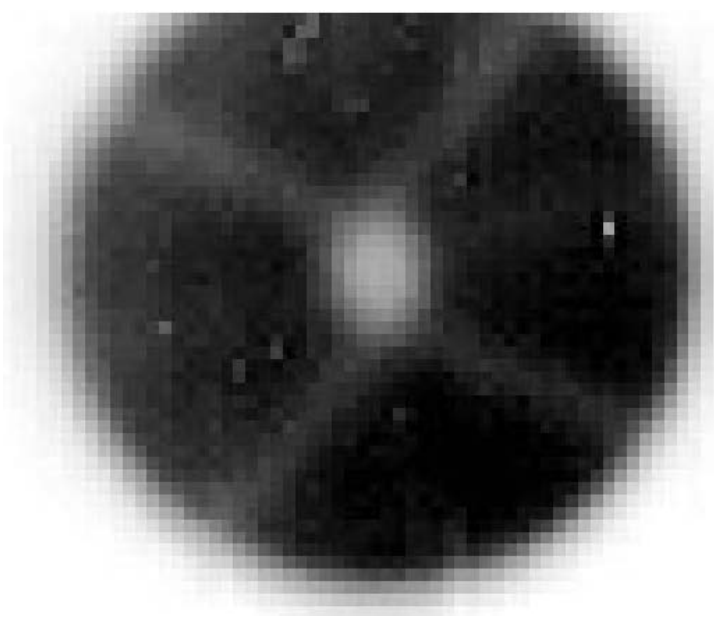

(b) Image of the pupil plane of MIDI obtained with UT3 in October 2003. Note the clear presence of the M2 mirror and the spiders holding this mirror. The read-out window for MIDI was not well centered on the image which left the upper part of the image unrecorded.

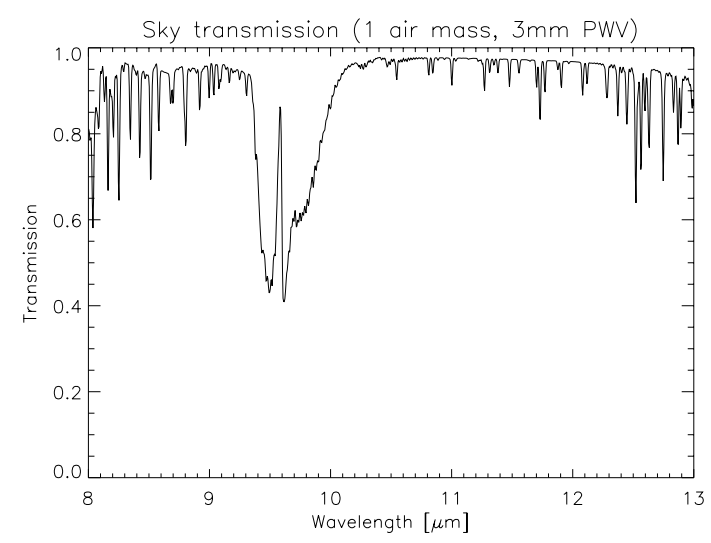

(c) Expected transmission of the atmosphere in the $\mathrm{N}$ band based on data obtained at Mauna Kea, taken from the Gemini web-site (http://www.gemini.edu/sciops/ObsProcess/).

Figure 1. Pupil plane images for MIDI and expected sky transmission of the $\mathrm{N}$ band for MIDI.

for a discrete signal:

$$
P=\sum_{j=0}^{N-1}\left|V_{j}\right|^{2}
$$

Noting $\widetilde{V_{k}}$ the Fourier transform of the time signal $V_{j}$ :

$$
\widetilde{V_{k}}=\sum_{j=0}^{N-1} V_{j} \exp ^{(-2 \pi i j k / N)}
$$


the power spectrum is given by:

$$
\operatorname{PSD}_{k}=\frac{1}{\Delta f}\left|\widetilde{V_{k}}\right|^{2}
$$

in units of $(\mathrm{ADU} / \mathrm{pixel} / \mathrm{sec})^{2} / \mathrm{Hz}$, with $\Delta f=\frac{1}{N \Delta t}$ in $\mathrm{Hz}$ and $k$ the index of the frequency axis of the power spectrum. In many cases the time series has been split in $M$ sub-series of each 100000 frames. In this case the PSDs are combined to a summed PSD $S_{k}$ following:

$$
S_{k}=\frac{1}{\Delta f M} \sum_{s=0}^{M}\left|\widetilde{V_{k}}\right|^{2}
$$

\subsection{Definition of spatial and spectral windows}

In order to reduce the amount of data to be handled we have defined several windows on the MIDI detector so as to keep only interesting regions. In Fig. 2 is shown the image of the detector with the definition of the spatial and spectral windows.

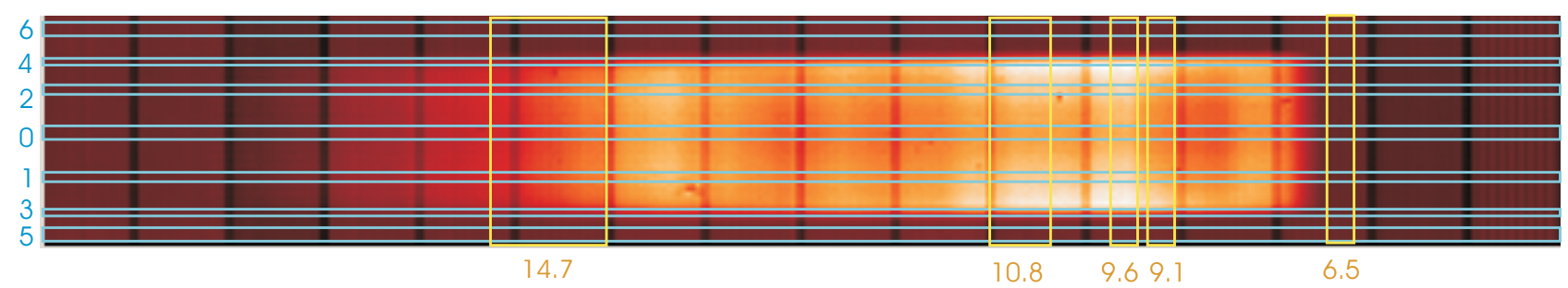

Figure 2. Image of the MIDI detector for one on-sky frame. Spectral information is coded in the horizontal direction (longest wavelength on the left side), while spatial information is in the vertical direction (image of the MIDI slit, 3 arcsecond long). Seven spatial windows have been defined, as well as five spectral windows.

\subsubsection{Spatial windows}

We have defined seven horizontal spatial windows, corresponding to five different positions in the sky plus two "off-sky" windows.

- Window \#0 is on-axis. It is the window in which the contribution of the sky background should be the largest and the contribution from the VLTI thermal emission the smallest;

- Windows \#1 and \#2 are slightly off-axis, at about 0.65 arcseconds from the optical axis. A significant increase of the background emission can be noticed in these windows due to thermal emission of the VLTI;

- Windows \#3 and \#4 are chosen at the edge of the slit image on the detector. At this location it is expected that even more VLTI thermal background falls onto the detector, at the expense of sky background;

- Windows \#5 and \#6 are located outside the slit image. They receive no background emission and are thus only affected by intrinsic detector noise.

\subsubsection{Spectral windows}

Five spectral regions have been selected for the analysis. Three of them are located within the $\mathrm{N}$ band, which is the nominal bandwidth for MIDI: these are the windows in which the sky emission is well transmitted through the instrument down to the detector. We have chosen two of these windows in the "clean" part of the sky spectrum $(8.82-9.34 \mu \mathrm{m}$ and $10.4-11.2 \mu \mathrm{m})$, as illustrated in Fig. 1(c), while the third one has been chosen inside the well-known ozone absorption line $(9.4-9.8 \mu \mathrm{m})$. This may allow to detect a different behavior of the sky background at this wavelength because of increased optical depth. Finally, we have defined two windows outside the MIDI bandpass $(6.34-6.73 \mu \mathrm{m}$ and $14.4-15.0 \mu \mathrm{m})$, where the sky emission is strongly attenuated by the low transmission of the instrument. These windows should be sensitive mainly to detector noise. 


\subsection{Spectrum of the background emission}

The raw data obtained during the October 2003 run consist in dispersed images of the background. Useful information on the background emission can already be deduced by comparing the spectra at different elevations and for different spatial windows. In Fig. 3 are illustrated the spectra of the background emission at elevations of $80^{\circ}$ and $50^{\circ}$ for spatial windows $\# 0,2,4$ and 6 .

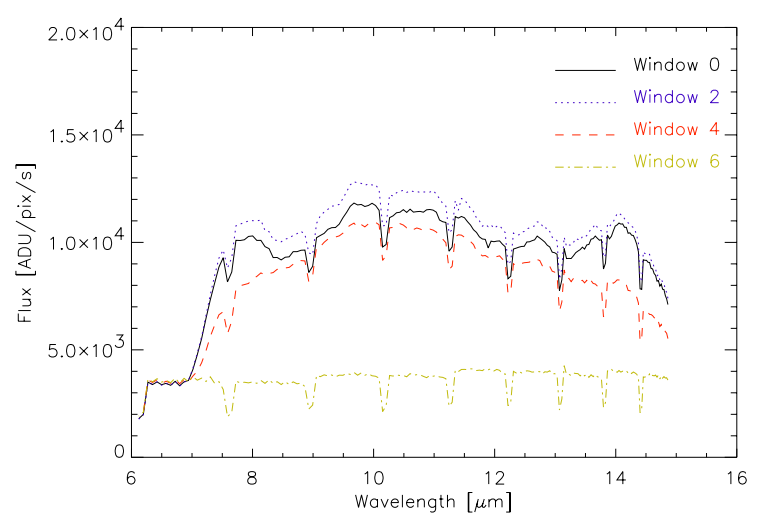

(a) $80^{\circ}$ elevation.

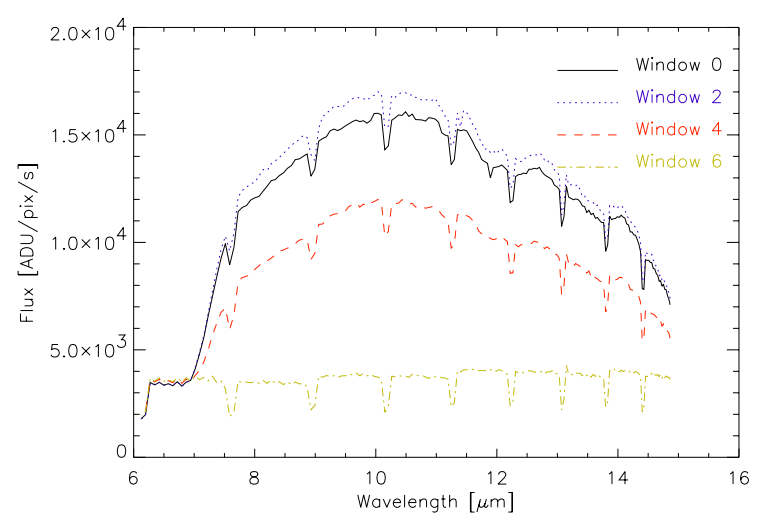

(b) $50^{\circ}$ elevation.

Figure 3. Cuts through the detector image for spatial windows $\# 0$, \#2, \#4 and \#6, at elevations $80^{\circ}$ (left) and $50^{\circ}$ (right). The spectral structure of the background emission for windows \#0 and $\# 2$ at $80^{\circ}$ are indicative of an important sky contribution, while window \#4 seems to be mainly affected by instrumental background (see text). At $50^{\circ}$, the three on-sky windows have the same spectral shape, indicative of a common background source (VLTI thermal emission).

At an elevation of $80^{\circ}$, the structure of the background emission changes between different spatial windows. The spectra for windows \#0 and \#2, which are quite similar, show a few characteristic features: the two bumps at 8 and $14 \mu \mathrm{m}$ are most probably associated to the edges of the atmospheric $\mathrm{N}$ band, at which the sky emission significantly increases, while the bump at $9.6 \mu \mathrm{m}$ is related to the contribution of the ozone layer, for which the optical thickness of the atmosphere is larger (see Fig. 1(c)). These bumps are only present in windows \#0 and \#2, meaning that these windows are more sensitive to sky emission, as expected. Window \#4, on the other hand, seems to be mainly affected by VLTI thermal emission, which is expected to have a smoother spectrum. Finally, window \#6 has a flat spectrum because it is not exposed to background light and is only affected by detector noise.

The bumps in the background spectrum are not present at low elevations (below $60^{\circ}$ ), for which the three on-sky windows have almost the same spectral shape. This suggests a common background source for these three windows. It is also noted that the level of background emission has significantly increased for windows \#0 and \#2 with respect to high elevations, while the level in window \#4 is almost the same as before. All these elements suggest that for some reason, the pupil re-imaging was worse at low elevations and that more VLTI infrastructure emission (and less sky emission) was sent to the center of the field-of-view. From this analysis of the background spectra, we conclude that the observations at high elevations (above $60^{\circ}$ ) are more sensitive to the sky emission than those at lower elevations.

\subsection{Power spectrum of the background emission}

During the technical run, long exposures of about 1 to 2 hours have been obtained on-sky with a sampling time of about 6 msec. From these series of detector images, we have produced time series for the 35 domains formed by the intersections of the seven spatial windows with the five spectral windows. The intensities recorded by all pixels inside each rectangular box have been averaged to produce the time series. From these time series, we have then produced PSDs for the background emission, which are presented and discussed in the next sections. 


\subsubsection{Power spectrum at high elevations $\left(>60^{\circ}\right)$}

In Fig. 4, we have plotted the PSD of the background emission registered at an elevation of $80^{\circ}$ in the four different types of spatial windows (namely, windows \#0,2,4 and 6) and for four different wavelengths. A few remarkable features are directly noticed in these PSDs:
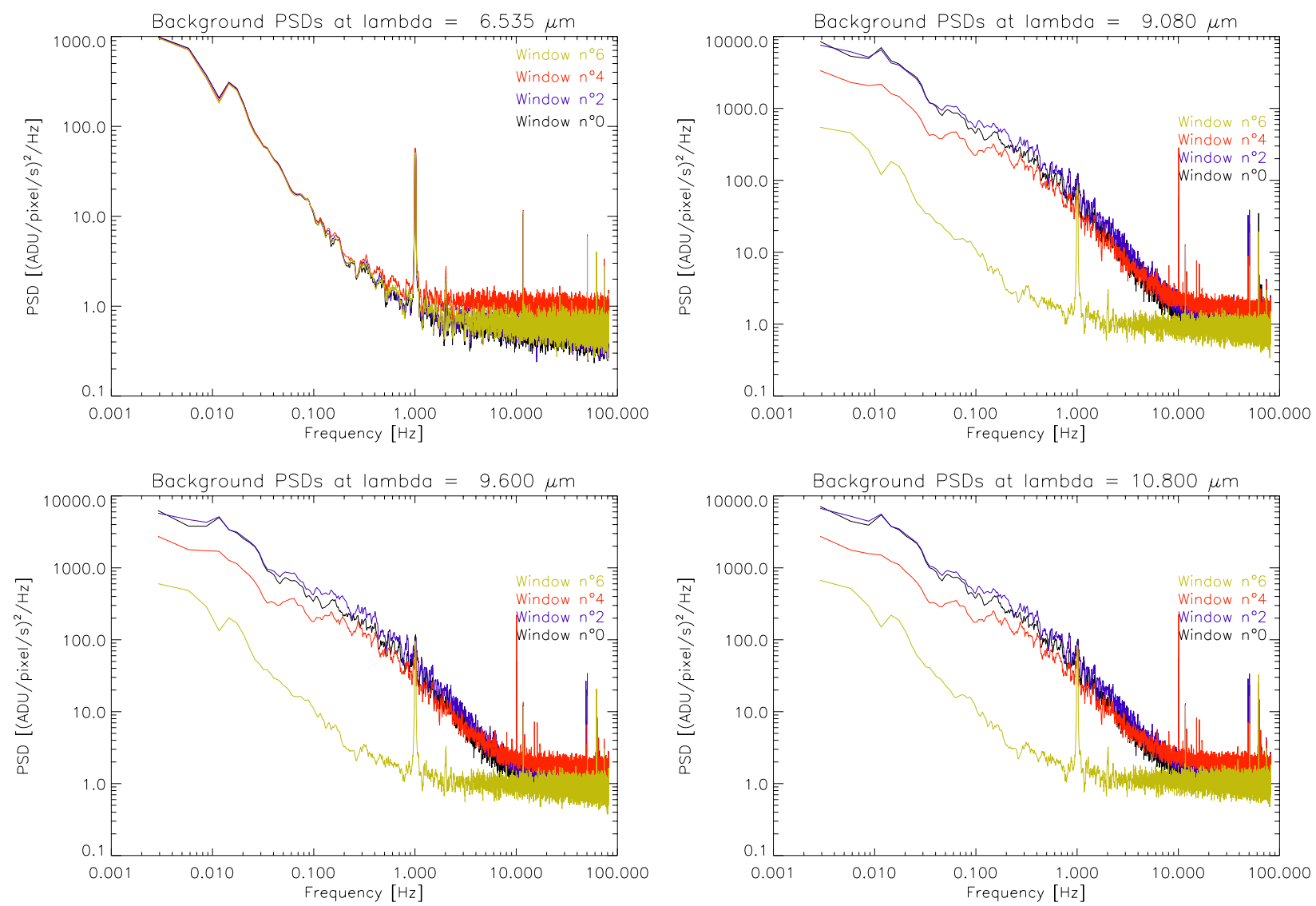

Figure 4. Power spectral densities (PSDs) of background fluctuations computed at an elevation of $80^{\circ}$ in the four selected spatial windows, for four different spectral windows.

- Detection noise is an important source of fluctuations, as illustrated by the PSDs taken in window \#6, which is not exposed to the sky and instrumental background. Spectral windows outside the MIDI bandwidth (i.e., outside the $\mathrm{N}$ band) are only affected by detection noise as expected. At high frequencies, the noise mainly consists in shot noise and has a flat (white) power spectrum, while, at low frequencies, fluctuations associated to the detector itself (drifts, etc) induce a large amount of noise in the recorded intensities. Detection noise can thus be approximated by a two-domain logarithmic power-law. At this particular elevation and moment in time, the slope of the power law at low frequencies is about -1.2 in logarithmic scale. For other measurements at high elevations, the recorded PSD had a log-log slope comprised between -1 and -1.7 ;

- The presence of background fluctuations is obvious at each of the three wavelengths inside the MIDI bandwidth: the PSDs of the three on-sky windows are largely above the PSD of the off-sky windows, in which only detector noise is present. This means that a large amount of fluctuation comes from the sky and/or instrumental background emission. Background fluctuations are dominant for frequencies below about $10 \mathrm{~Hz}$, and can be described by a power-law with a logarithmic slope of about -1 ; 
- Among the on-sky windows, window \#4 has a slightly lower level of fluctuation. As window \#4 is expected to be more sensitive to VLTI thermal emission and less sensitive to actual sky background, this indicates that the fluctuations are mainly associated to the sky background and not to the instrumental one. This will be confirmed below when discussing the PSDs at lower elevations;

- Several peaks can be observed in the PSDs. They are related to instrumental effects and will be discussed in section 3.5.

\subsubsection{Power spectrum at low elevations $\left(<60^{\circ}\right)$}

In Fig. 5 are plotted the PSDs for an elevation of $50^{\circ}$. Inside the $\mathrm{N}$ band, the PSDs of the on-sky windows are still above the detection noise, but their level has strongly decreased. The contribution of actual background fluctuations to the total noise is only noticeable between about 0.1 and $2 \mathrm{~Hz}$ and is only slightly larger than detection noise. In this frequency range, it can be fitted with a power law of logarithmic slope -0.8 . Since we have shown in section 3.3 that most of the background emission is due to VLTI thermal emission at low elevation, and consequently that the level of sky background is much smaller, this suggests once again that instrumental background is much more stable than the sky background. The large fluctuations observed at high elevations are thus well due to the sky background itself.
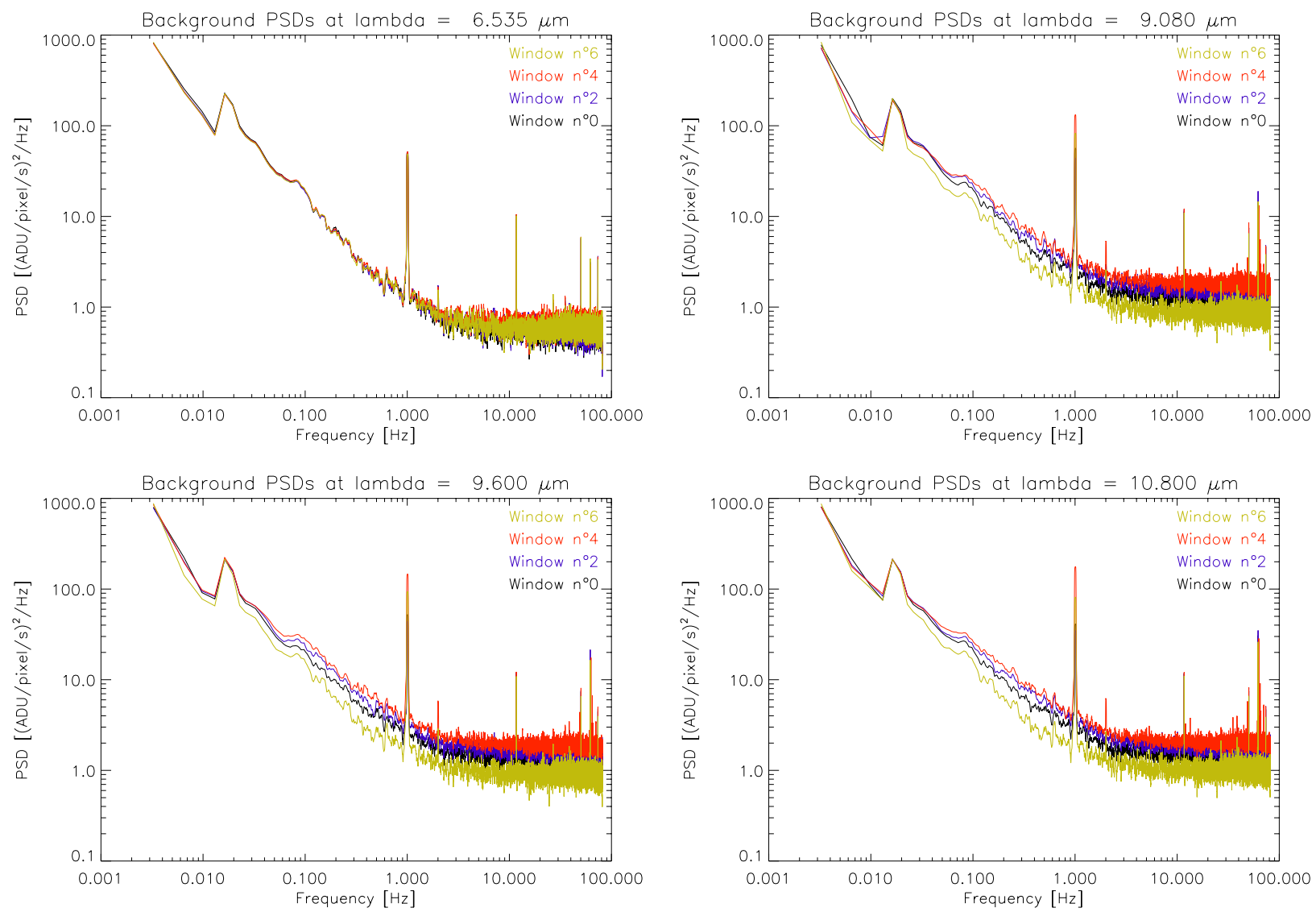

Figure 5. Power spectral densities (PSDs) of background fluctuations computed at an elevation of $50^{\circ}$ in the four selected spatial windows, for four different spectral windows.

It can also be noticed that, both at $80^{\circ}$ and $50^{\circ}$, the PSDs for the three spectral windows inside the $\mathrm{N}$ band are pretty similar. Indeed, background fluctuations show no evidence of wavelength dependence across the $\mathrm{N}$ 
band. The slope and the precise shape of the PSDs are almost identical in these three spectral windows. There seems to be no influence from the ozone layer on the power spectrum either. Whether this behavior can be extended towards shorter wavelengths is of course debatable, but in first approximation the same slope for the power-law of background fluctuations could be used for the GENIE simulations in the L' band. This would also be in agreement with near-infrared measurements of the sky noise carried out in Siding Springs (Australia) in 1981 (see Ref. 3), for which the measured slope of the power-law was about -1.3 in the L band.

\subsubsection{Discussion and consequences for VLTI mid-IR instruments}

On the average a slope of -1 to almost -2 is measured for the log-log plot of power versus frequency. This is less steep than a Kolmogorov spectrum (slope is -8/3) and that for a random walk signal $(-2)$. We should of course realize that our measurements were made in the image plane and therefore averages intensity fluctuations which exist over the pupil plane are note directly measured, and even averaged. This is consistent with a slope flatter than -2 (Fig. 6).

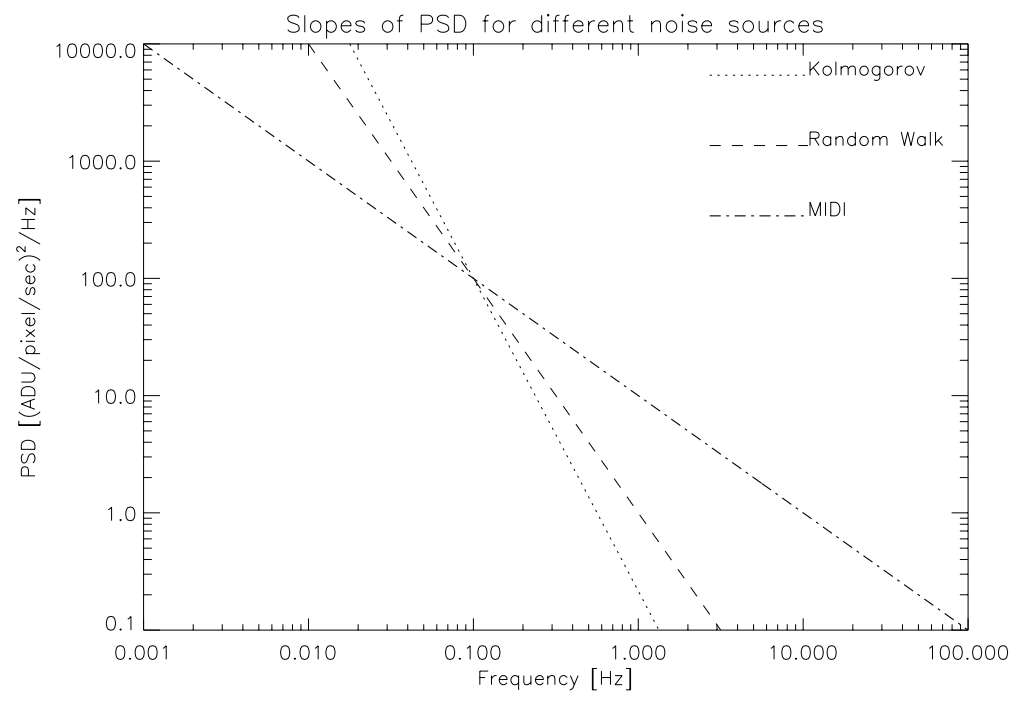

Figure 6. Comparison of the slope for different sources of statistics: Kolmogorov, random walk, and as measured with MIDI in the $\mathrm{N}$ band.

In order to study the effects of thermal background fluctuations on the performance of mid-infrared instruments, measuring the background characteristics in the image plane is the relevant method. On the other hand, if one wishes to study the distribution of background emission on the sky, measurements in the pupil plane might be more useful.

The PSDs displayed here show that the transition between fluctuation-limited and photon noise-limited regimes occur around $10 \mathrm{~Hz}$. This is thus the typical frequency that should be used by the VLTI instruments in order to operate in background-limited performance (BLIP) range. In the case of GENIE, BLIP is not sufficient: the background emission must be calibrated with a very high accuracy (typically $10^{-4}$ to $10^{-5}$ for an $\mathrm{L}^{-}$-band nulling instrument). Let us try to estimate at which frequency chopping should be carried out in order to reduce the residual background fluctuations below a relative level of $10^{-4}$. For this estimation, we will assume that a slope of about -1.5 in log-log scale is valid for the PSD of background fluctuations up to an arbitrary high frequency. This assumption is of course debatable since our data set does not show what happens to background fluctuations at frequencies above $10 \mathrm{~Hz}$, and also because the extrapolation of the PSD observed in the N band towards the L' band is not fully justified. It might well be that the slope becomes steeper at higher frequencies (e.g. because of pupil averaging), which would make the following estimation pessimistic.

We have chosen an uninterrupted on-sky time series of $10 \mathrm{~min}$ at an elevation of $80^{\circ}$ to compute the mean and standard deviation of the background emission, at a wavelength of $9.6 \mu \mathrm{m}$ in dispersed mode. A mean 
photon rate of $1.7 \times 10^{6} \mathrm{ph}-\mathrm{el} / \mathrm{pix} / \mathrm{sec}$ was found, with a standard deviation of $2.8 \times 10^{3}$. This standard deviation contains both the contribution of the photon noise and of the fluctuation noise. Since photon noise is given by the square root of the mean photon arrival rate, we can compute the contribution of fluctuation noise as:

$$
\operatorname{RMS}_{f l u c}=\sqrt{\left(2.8 \times 10^{3}\right)^{2}-1.7 \times 10^{6}},
$$

which gives $\mathrm{RMS}_{\text {fluc }} \simeq 2.5 \times 10^{3} \mathrm{ph}$-el/pix/sec for the given frequency range $\left(f>3.3 \times 10^{-3}\right.$ for a 10 -min time series). This represents a fraction of $10^{-3}$ of the mean background emission. The variance of the residual background fluctuation for a chopping frequency $f_{c}$ is given by the integral of the PSD for frequencies above $f_{c}$, and can be computed as follows:

$$
\begin{aligned}
\int_{f_{c}}^{\infty} \alpha f^{-1.5} \mathrm{~d} f & =2 \alpha f_{c}^{0.5} \\
& =\left(\frac{f_{c}}{3.3 \times 10^{-3}}\right)^{0.5} \int_{3.3 \times 10^{-3}}^{\infty} \alpha f^{-1.5} \mathrm{~d} f
\end{aligned}
$$

with $\alpha$ a normalization factor for the PSD of background fluctuation ( $\left.\mathrm{PSD}=\alpha f^{-1.5}\right)$ and with the last integral having its square root equal to $2.5 \times 10^{3} \mathrm{ph} / \mathrm{pix} / \mathrm{sec}$ (this is the standard deviation we have just computed above). In order to reach a precision of $10^{-4}$ in background subtraction, a chopping frequency of about $150 \mathrm{~Hz}$ must be used. The precision in background correction increases very slowly, proportionally to $f_{c}^{0.25}$, with our (pessimistic) assumption of a -1.5 slope up to an arbitrary high frequency. Classical chopping with the M2 mirror of the VLTI cannot be performed at such a high frequency, which implies that a more advanced chopping technique, allowing higher chopping frequencies, should be used for GENIE.

\subsection{Instrumental effects}

Let us briefly discuss the origin of the numerous peaks present in the PSDs of background fluctuations.

- Peaks at 1, 2 and $3 \mathrm{~Hz}$ are attributed to the MIDI closed-cycle cooler. The liquid helium expands close to the detector's cold finger. This process slightly modifies the temperature of the detector and hence the efficiency. This leads to a modulation in the detected intensity. A replica of this peak can be observed at $2 \mathrm{~Hz}$, and sometimes even at $3 \mathrm{~Hz}$;

- The strength of the $10 \mathrm{~Hz}$ peak seems to be correlated to the strength of the background fluctuations (peak is higher at high elevations) and only present in the off-axis PSD, which means that it is directly affecting the light beams, upstream the detector. Indeed, this peak is very intense for window \#4, located at the edge of the slit, and almost absent from window \#0 at the center of the slit. These two correlations seem to fit the model that something with the VLTI infrastructure partially blocks the sky light, and has a $10 \mathrm{~Hz}$ modulation. Since the vignetting increases with the elevation (probably an effect of the alignment of the beam) this peak is stronger for larger elevation, and also stronger for detector pixels which are more off-axis;

- Peaks at 30.1-30.3 Hz, as well as peaks at 62.3 and $74.0 \mathrm{~Hz}$ occurred consistently on one of the observing runs, but not on both. The most likely explanation is that the optical train was different, and that these frequencies are associated with modulation by components of the optical train;

- The $50 \mathrm{~Hz}$, which is observed in both observing runs, is also observed for the experiments where a black-plate was inserted into the beam and also those which looked at the black-screen just in front of the detector. This suggest that the $50 \mathrm{~Hz}$ comes from the detector, and most likely from the read-out electronics. Alternatively other equipment may generate fields that effect the detector signal going through the MIDI cables. 
Table 2. Census of the instrumental peaks present in the PSDs of the data acquired. If the peak is not always observed at exactly the same frequency but has a certain range of occurrence, it is presented in the frequency column as a range of frequencies. Rate of occurrence is represented by asterisks, each asterisk representing $10 \%$ of the total sample. In addition to the peaks listed hereunder, a large number of peaks $(16.0,16.9,18.0,24.2,26.0,27.0,36.0,53.5,55.1$ and $71.6 \mathrm{~Hz}$ ) were observed when engineers were present near the Coudé platform to conduct engineering tasks.

\begin{tabular}{|c|c|c|c|c|}
\hline $\begin{array}{c}\text { Frequency } \\
{[\mathrm{Hz}]}\end{array}$ & $\begin{array}{l}\text { Run } 1 \\
\text { Feb.03 }\end{array}$ & $\begin{array}{l}\text { Run } 2 \\
\text { Oct.03 }\end{array}$ & Occurrence & Origin \\
\hline $\begin{array}{c}1.0 \\
2.0 \\
3.0 \\
10.0-10.2 \\
11.8 \\
30.1-30.4 \\
42.5 \\
47.3 \\
48.6 \\
50.0-50.4 \\
62.3 \\
69.2-69.8 \\
74 \\
79.7-80.6 \\
83.5-83.6\end{array}$ & $\begin{array}{l}\mathrm{y} \\
\mathrm{y} \\
\mathrm{y} \\
\mathrm{y} \\
\mathrm{y} \\
\mathrm{y} \\
\mathrm{y} \\
\mathrm{y} \\
\mathrm{y}\end{array}$ & $\begin{array}{l}\mathrm{y} \\
\mathrm{y} \\
\mathrm{y}\end{array}$ & $\begin{array}{c}* * * * * * * * * \\
* * * * * * * * \\
* \\
* * * * * \\
* * * \\
* * * * * \\
* * \\
* * * \\
* * * * * \\
* * * * * * * * * \\
* * * * * \\
* * * * * \\
* * * * * \\
* * * * \\
* * * *\end{array}$ & $\begin{array}{l}\text { Closed-cycle cooler } \\
\text { Replica of the } 1 \mathrm{~Hz} \text { peak } \\
\text { Only on } 1 \text { event } \\
\text { Infrared emission from components in the optical path } \\
\text { Only for February 2003, not for October } 2003 \\
\text { Power line frequency } \\
\text { Only for October } 2003, \text { not for February } 2003 \\
\text { Only for October } 2003, \text { not for February } 2003\end{array}$ \\
\hline
\end{tabular}

\subsection{Spatial correlations}

Thanks to the spatial extent of the MIDI slit, of about 3 arcseconds, we can investigate the spatial structure of the background emission. We have already seen that the emission peaks at the edges of the slit, where more VLTI thermal emission makes it into the field-of-view. This is illustrated in Fig. 7, where we have plotted the intensity of the background emission as a function of spatial position (i.e., along the vertical direction on the detector). This figure also illustrates that the structure of the background emission is flatter at lower elevations, which could also be attributed to more VLTI thermal emission falling onto the detector.
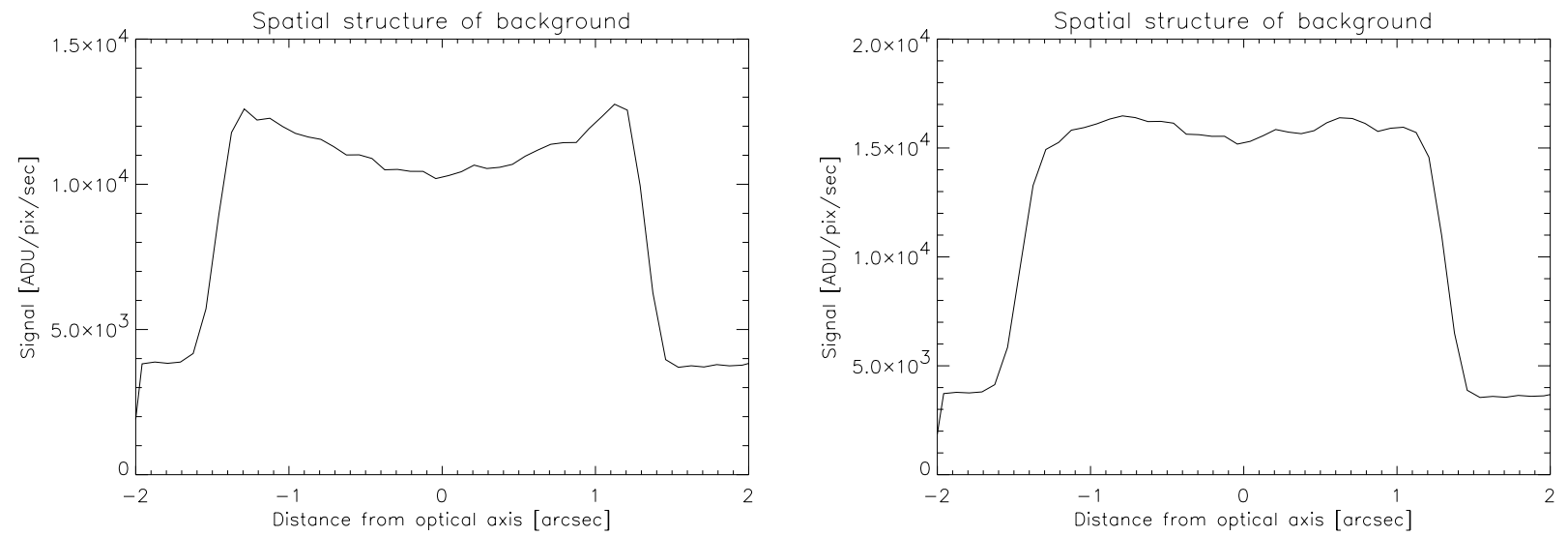

Figure 7. Spatial structure of the background emission, plotted along the slit direction for a wavelength of $9.5 \mu \mathrm{m}$, respectively at elevations of $80^{\circ}$ (left) and $50^{\circ}$ (right). 

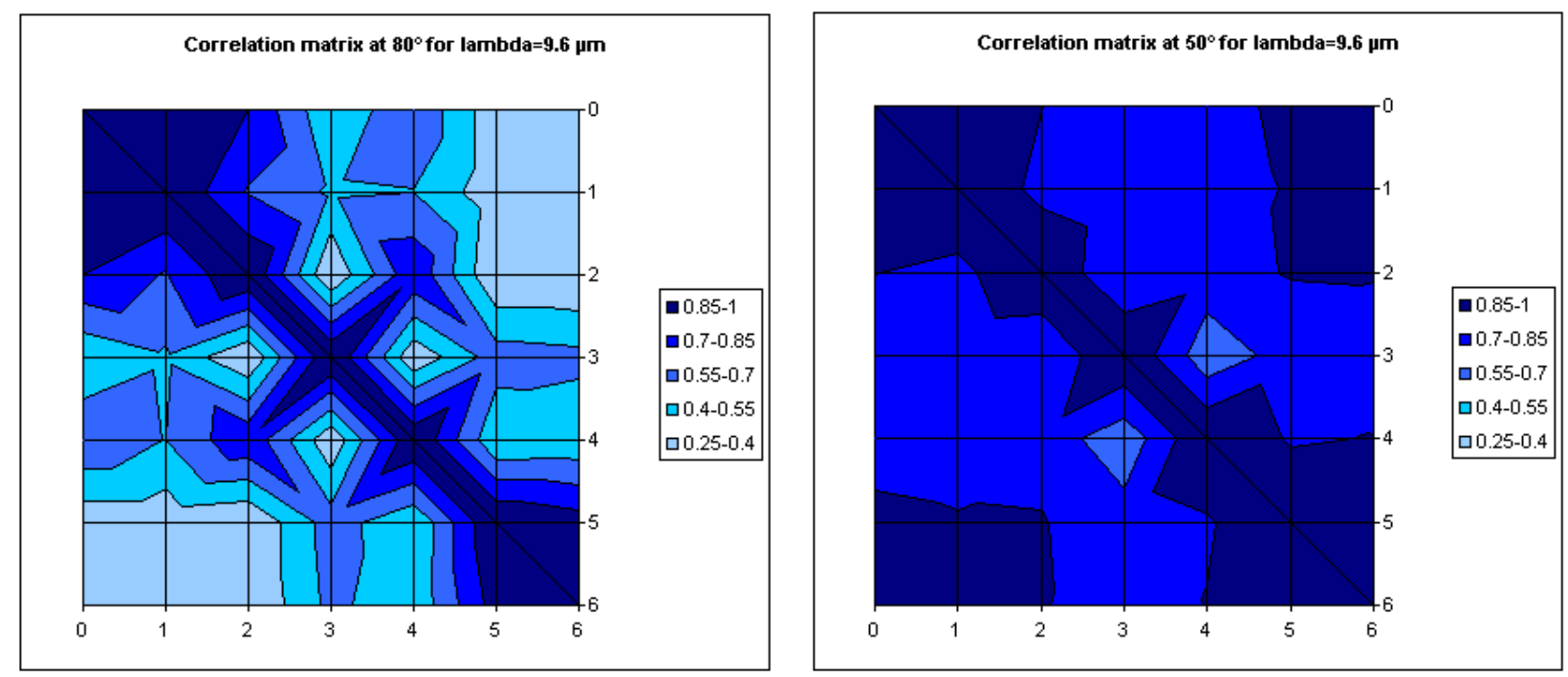

Figure 8. Study of correlation between time series for background emission in different spatial windows (as defined in Fig. 2), respectively at elevations of $80^{\circ}$ (left) and $50^{\circ}$ (right).

In Fig. 8 we have computed the correlation between the time series for background fluctuations between different spatial windows, at a wavelength of $9.6 \mu \mathrm{m}$ and for two different elevations. This figure shows that the correlation between spatial windows is much larger at low elevations. This indicates that a common source of background emission affects all spatial windows, and once again suggests VLTI thermal emission to be the main contributor to the background at low elevations. Fig. 8 also shows that a higher correlation exists between spatial windows close together, as expected. For instance, the correlation between windows \#0, 1 and 2 is quite high, while the correlation between windows \#1 and 4, \#2 and 3 or \#3 and 4 is rather low (especially at high elevations). This means that some loss of correlation is already detectable in the background emission for an angular distance of 1 arcsecond, which would suggest classical chopping methods to use rather small off-axis angles for background measurements. However, the presence of more VLTI thermal emission in off-axis windows might be the actual reason for this loss of correlation. This statement should thus be refined with further sky background measurements when the Variable Curvature Mirrors come on-line and allow for a clean pupil re-imaging inside the MIDI instrument.

\section{CONCLUSIONS}

In this paper, we have characterized the fluctuations of the thermal background emission at the VLTI through its power spectral density. The PSD of background fluctuations can be described in terms of a broken power law with two regimes:

- a slope generally comprised between about -1 and -1.5 in $\log -\log$ scale is identified at low frequencies $(f<10 \mathrm{~Hz})$;

- a flat power spectrum is observed at frequencies above $10 \mathrm{~Hz}$, characteristic of pure photon noise.

There are strong indications that the fluctuations at low frequencies are mainly due to the turbulence of the atmosphere outside the VLTI infrastructure.

In addition to the two-domain power law, a series of narrow peaks have been observed in the PSDs. These peaks are suspected to be due to instrumental effects, such as the $1 \mathrm{~Hz}$ cycle of the MIDI close cycle cooler, the $50 \mathrm{~Hz}$ frequency of the power line, as well as some mechanical vibrations in the VLTI optical train at various frequencies. The results are however limited by the unavailability of a pupil re-imaging device inside the VLTI 
delay lines, which caused some spurious thermal light emitted by the VLTI infrastructure to reach the detector and add a significant contribution to the recorded background emission. Moreover, during the February 2003 run, the beams from UT1 and UT3 were vignetted, so receiving even more radiation from the infrastructure.

From this study, we are able to give a few recommendations for the chopping parameters to be used with the VLTI mid-infrared instruments. In order to reach background-limited performance with MIDI, a chopping frequency of about $10 \mathrm{~Hz}$ should be used, and in order to sample spatially correlated background regions in the sky, the chopping stroke should not be too large (not more than a few arcseconds*). These two results are in good agreement with a previous study by Käufl et al. (Ref. 4). In the case of GENIE, where a higher precision on background subtraction is mandatory, a higher frequency is needed for chopping (about $150 \mathrm{~Hz}$ according to our estimations), requiring a more advanced chopping method than classical chopping with the VLTI M2 mirror.

\section{ACKNOWLEDGMENTS}

O. A. acknowledges the financial support of the Belgian National Fund for Scientific Research. E. J. B. is supported by NEVEC, a project of NOVA, the Netherlands Research School For Astronomy. The authors would like to thank the MIDI consortium (principal investigator: Christoph Leinert; project manager: Uwe Graser; software manager: Walter Jaffe, co-PI: Rens Waters, Guy Perrin) to make this experiment possible.

\section{REFERENCES}

1. C. Leinert, U. Graser, L. B. F. M. Waters, G. Perrin, W. Jaffe, B. Lopez, F. Przygodda, O. Chesneau, P. A. Schüller, A. W. Glazenborg-Kluttig, W. Laun, S. Ligori, J. A. Meisner, K. Wagner, E. J. Bakker, B. Cotton, J. A. De Jong, R. J. Mathar, U. Neumann, and C. Storz, "Ten-micron instrument MIDI: getting ready for observations on the VLTI," in Interferometry in Optical Astronomy II, W. Traub, ed., Proc. SPIE 4838, pp. 893-904, 2003.

2. P. Gondoin, O. Absil, C. V. M. Fridlund, C. Erd, R. H. Den Hartog, N. Rando, A. Glindemann, B. Köhler, R. Wilhelm, A. Karlsson, L. Labadie, I. Mann, A. J. Peacock, A. Richichi, Z. Sodnik, M. Tarenghi, and S. Volonte, "Darwin ground-based European nulling interferometer experiment (GENIE)," in Interferometry in Optical Astronomy II, W. Traub, ed., Proc. SPIE 4838, pp. 700-711, 2003.

3. D. A. Allen and J. R. Barton, "A study of sky noise, 1.5-5 microns," Astronomical Society of the Pacific, Publications 93, pp. 381-384, June-July 1981.

4. H. U. Käufl, P. Bouchet, A. Van Dijsseldonk, and U. Weilenmann, "A sky-noise measurement and its implication for ground-based infrared astronomy in the $10 \mu \mathrm{m}$ atmospheric window," Experimental Astronomy 2(2), pp. 115-122, 1991.

5. R. Wilhelm and P. Gitton, "The VLTI environment and GENIE," in Toward Other Earths: Darwin/TPF and the Search for Extrasolar Terrestrial Planets, M. Fridlund and T. Henning, eds., ESA SP-539, pp. 659-664, ESA's Publication Division, 2003. ISBN 92-9092-849-2.

6. D. F. Buscher, J. T. Armstrong, C. A. Hummel, A. Quirrenbach, D. Mozurkewich, K. Johnston, C. S. Denison, M. M. Colavita, and M. Shao, "Interferometric seeing measurements on Mt. Wilson: power spectra and outer scales," Applied Optics 34, pp. 1081-106, February 1995.

\footnotetext{
${ }^{*}$ This statement is still subject to caution because of the bad pupil re-imaging encountered during our technical runs.
} 